\title{
Predicting and controlling infectious disease epidemics using temporal networks Naoki Masuda ${ }^{1 *}$ and Petter Holme ${ }^{2,3,4}$
}

\author{
Addresses: ${ }^{1}$ Department of Mathematical Informatics, The University of Tokyo, 7-3-1 Hongo Bunkyo, Tokyo 113-8656, Japan; \\ ${ }^{2}$ Department of Energy Science, Sungkyunkwan University, Suwon 440-746, Korea; ${ }^{3}$ IceLab, Department of Physics, Umeå \\ University, 90187 Umeå, Sweden; ${ }^{4}$ Department of Sociology, Stockholm University, 10691 Stockholm, Sweden \\ *Corresponding author: Naoki Masuda (masuda@mist.i.u-tokyo.ac.jp) and Petter Holme (holme@skku.edu) \\ FI000Prime Reports 2013, 5:6 (doi:10.12703/P5-6) \\ This is an open-access article distributed under the terms of the Creative Commons Attribution-Non Commercial License \\ (http://creativecommons.org/licenses/by-nc/3.0/legalcode), which permits unrestricted use, distribution, and reproduction in any medium, \\ provided the original work is properly cited. You may not use this work for commercial purposes. \\ The electronic version of this article is the complete one and can be found at: http://f1000.com/prime/reports/b/5/6
}

\begin{abstract}
Infectious diseases can be considered to spread over social networks of people or animals. Mainly owing to the development of data recording and analysis techniques, an increasing amount of social contact data with time stamps has been collected in the last decade. Such temporal data capture the dynamics of social networks on a timescale relevant to epidemic spreading and can potentially lead to better ways to analyze, forecast, and prevent epidemics. However, they also call for extended analysis tools for network epidemiology, which has, to date, mostly viewed networks as static entities. We review recent results of network epidemiology for such temporal network data and discuss future developments.
\end{abstract}

\section{Introduction}

Infectious diseases are a major threat to public healthestimated to account for $43 \%$ of the global burden of disease (where the burden of health is measured in the number of years of healthy life lost http://www.who.int/ trade/glossary/story036/en/). To mitigate their spreading, we need to understand pathogenesis, environmental factors, and the social structure of contagion. Network theory has become a valuable framework to study the last one-the role of the contact patterns in epidemics. Social networks among individuals (i.e. humans and animals), as the one shown in Fig. 1 affect the possibility, extent, and speed of epidemic spreading. Lately, contact pattern data with precise temporal information of the contacts (i.e., timing and duration of contacts) between individuals have been collected in various situations. The aim in this report is to discuss the relevance of such data, collectively called the temporal networks $[1,2]$, to theoretical epidemiology.

Network epidemiology has proved an indispensable approach for understanding epidemics of infectious disease, often acknowledged in medical epidemiology, mathematical biology, and more recently network science [3-15]. A recent practical example of network epidemiology is the
GLEAMviz platform that succeeded in forecasting the 2009 H1N1 pandemic [16] (also see $[17,18]$ ). An implicit assumption behind most network epidemiology studies is that a link (i.e., contact) between two individuals is an incessant pathway for contagion. This is clearly a strong simplification of reality. In fact, data possessing precise temporal information of the events of a (potentially contagious) contact between individuals, collected from humans and other animals, are available nowadays. In a temporal network approach to such datasets, we view a link as dynamic entity and assume that contagion can occur only inside the time window in which the two individuals interact. With this view in mind, the static approach of network epidemiology may miss a great deal of what is happening in reality.

An early example of such temporal network epidemiology is found in the analysis of sexually transmitted infections (STIs). First of all, STIs lend themselves naturally to network epidemiological approaches because a contact is welldefined and in many cases stable on the timescale of the epidemics. In a conventional survey, one asks respondents about the sexual partners over some certain time frame, often spanning several years, to generate a static sexual 


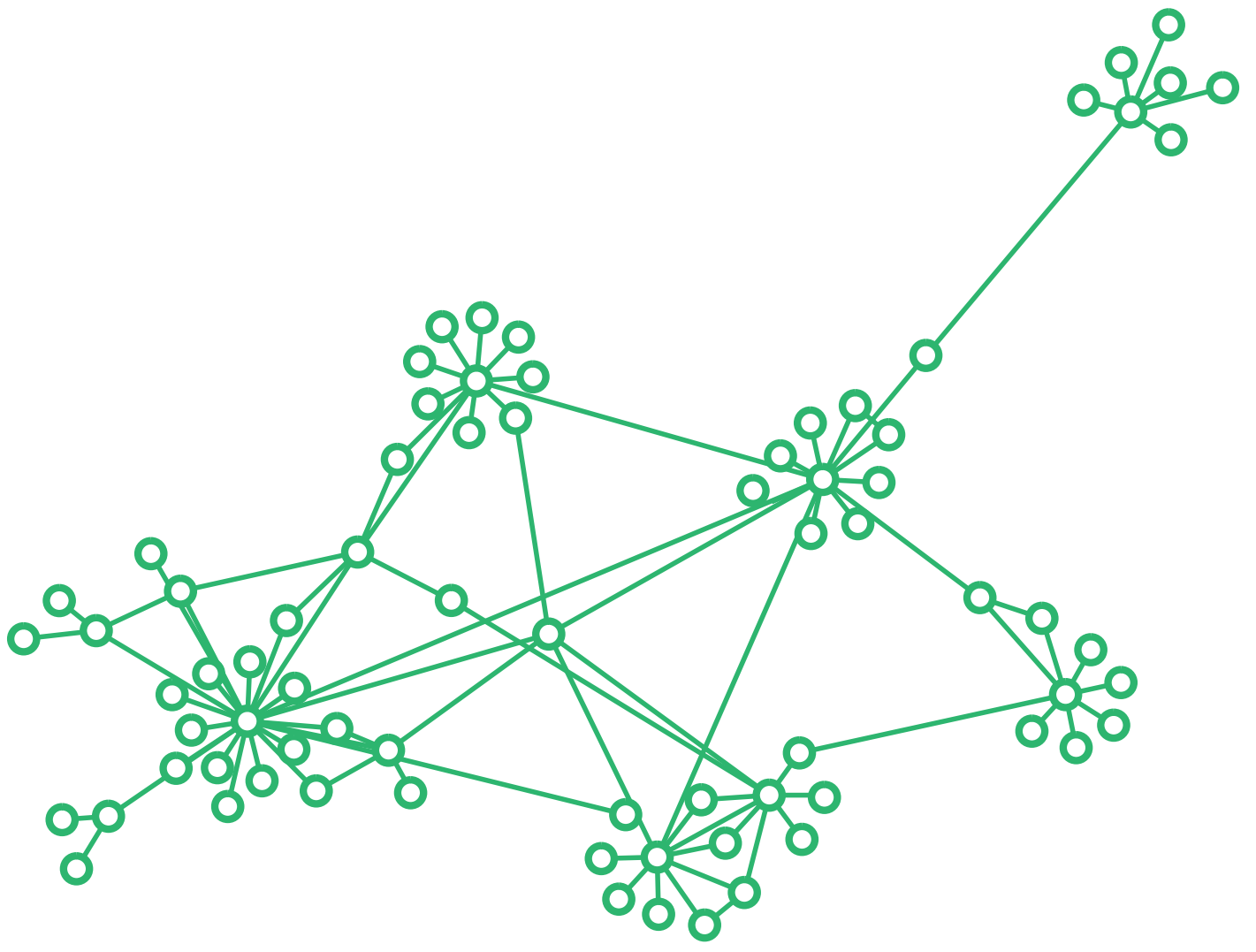

The network of sexual contacts between Icelandic men. Data from [19]. More specifically, this is the network of contacts confirmed by both the actors.

contact network [19-22]. However, if we consider the temporal aspect of contacts of a given individual, a disease can be transmitted from an old partner to a new partner, via the focal individual, but not vice versa [21,23-25]. An early attempt to account for the difference between sexual contact patterns-like people being serial monogamists or having concurrent partnerships-was the concurrency measure proposed by Kretzschmar and Morris [26,27]. This measure is based on the network of contacts that have happened by a given time. Because the concurrency measure is based on a network of accumulated contacts, it does not incorporate all the temporal information that a temporal network could. Nevertheless, the philosophy behind it rests on a temporal network thinking and thus Kretzschmar and Morris's work is a precursor of temporal network epidemiology.

As mentioned above, these days we have access to an increasing amount of temporal network data. The most common instance is data of online social contacts [28-31], although it is perhaps not directly related to epidemic spreading (other than computer and email viruses).
Another temporal network data source of human communication that people have investigated is mobile phone calls [25,32-37]. Yet more relevant, as it logs actual physical proximity (and thus potential disease-spreading events), is data recorded from humans wearing Bluetooth sensors of mobile phones [38-40], Radio-Frequency Identification Devices [41,42], and infrared modules [43-46]. In particular, the SocioPatterns project (http:// www.sociopatterns.org/) [41,42] collected data from a community hospital [47] and a primary school [48], where epidemic spreading is an important issue. On a slower timescale, airport networks, which mediate global epidemic outbreaks, are also dynamic [49]. Pertaining to infections of animals, data obtained from, for example, sheep [50], cattle [51-53], ants [54], and zebra [55] have been analysed in different contexts. As mentioned, this is probably only the beginning-we will have access to an increasing number of such temporal network datasets in the near future. Then, we will also need a temporal network approach to understand the role of contact patterns in epidemics. By exploiting the information in temporal networks, we can better describe, predict, and 
control epidemics than by only relying on static-network epidemiology. Essentially, ignoring temporal correlations, as a static network approach does, can lead to an over- or underestimation of the speed and extent of an outbreak. Notwithstanding, temporal-network epidemiology, both analytical and computational, is still in its infancy, and more powerful methods are urgently needed. We will expand on this topic and discuss emergent issues and unsolved problems. We focus on the following two specific problems in this report.

\section{Effects of temporal networks on epidemic spreading and the need of developing analysis methods}

Recent analysis, mainly numerical, showed that statistics of outbreaks (such as their final size and speed of spreading) differ considerably between empirical temporal networks and corresponding static networks. However, how and when they are different is not sufficiently understood. We describe the results of some key studies, compare them, and identify the problems inherent in the current temporal network approaches to epidemics.

\section{Disease prevention methods}

A basic issue in epidemiology, both in theory and practice, is to develop methods to mitigate disease spreading by immunizing individuals or restrict their interaction with others. By exploiting temporal information of the contacts, it may be possible to contrive disease prevention strategies that outperform existing ones. We describe very recent approaches in this direction (there are only a few papers though) and suggest where this kind of study should be directed.

Our main thesis is that the use of temporal contact networks makes it possible to better describe, predict, and control epidemic spreading in many practical situations. In the first main section, we describe temporal networks and illustrate differences between them and the conventional static networks. In the second main section, we briefly introduce mathematical epidemic models that are often used in theoretical and numerical studies of epidemic spreading on networks. The third main section describes recent results on epidemic spreading on temporal networks. The fourth main section is devoted to (preliminary studies of) prevention measures on temporal networks. Finally, we will discuss unsolved issues and future directions.

\section{Temporal networks}

From a static social-network viewpoint, a link between a pair of individuals usually indicates that the two individuals interact at least once within a sampling period. The collection of links and the participating individuals form a network (Fig. 1 shows an example). Epidemic spreading on networks has been an intensively investigated topic involving different research fields [3-15]. In this section, we will discuss some interesting examples.

The temporal network is an emerging modelling framework for understanding epidemic spreading on social networks [1,2]. The "atoms" making up a temporal network are the contacts (sometimes called events). A contact is the interaction between two individuals such that a disease can spread from one to another. The contact is either treated as lasting for a certain amount of time or as instantaneous. A link may contain multiple contacts between the same pairs of individuals occurring at different times. In fact, different contacts on the same link may have different impacts on dynamics on networks and hence on epidemic spreading [46]. In addition to the time, the duration of contact is an important component of a temporal network that affects epidemic spreading. Peer-to-peer infection would occur if the contact has a long duration.

An example of a temporal network is depicted in Fig. 2(A) and even this small example clearly shows the importance of considering how networks vary with time. In Fig. 2(A), an infectious disease may spread from individual A to D via B. However, the converse cannot occur. This is because there is at least one "temporal path" from A to D but not vice versa [1,56-58].

If we disregard the timing information in the temporal network shown in Fig. 2(A), we obtain the static, aggregated network shown in Fig. 2(B). If we simulate an outbreak on the aggregated network, a chain of infection from A to D may occur, and vice versa. This is because the static network shown in Fig. 2(B) is undirected (i.e., only bidirectional links are assumed), which corresponds to the fact that the contacts in Fig. 2(A) are implicitly assumed to be bidirectional. There is no way of encoding all the information contained in Fig. 2(A) into Fig. 2(B). The lesson here is that we may be misguided if we only use aggregated static networks. One may argue that a real temporal network lasts much longer than that shown in Fig. 2(A), so that temporal paths could exist between any ordered pair of individuals to provide pathways for pathogens. In fact, this is generally true. However, even in such a case, the likelihood with which a chain of infection from $\mathrm{A}$ to $\mathrm{D}$ occurs and that of the inverse chain (i.e. from $\mathrm{D}$ to A) are usually different. Furthermore, the fact that pathogens may mutate or other factors affecting the disease spreading may change on a fairly short timescale is a reason to restrict the sampling time.

Another well-documented phenomenon that occurs in real temporal networks and is relevant to epidemiology is the "bursty" nature of events (i.e. a mixture of short and 
Figure 2. Temporal network and its aggregated counterpart

(A)

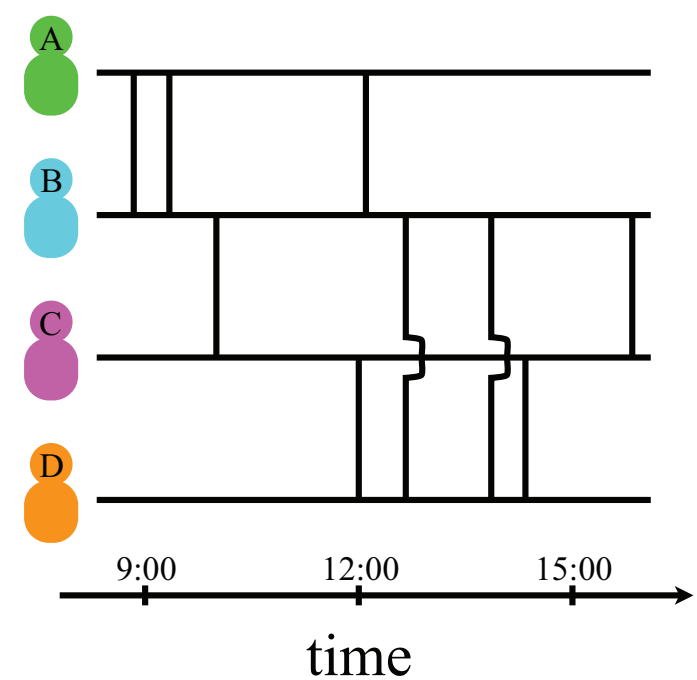

(B)
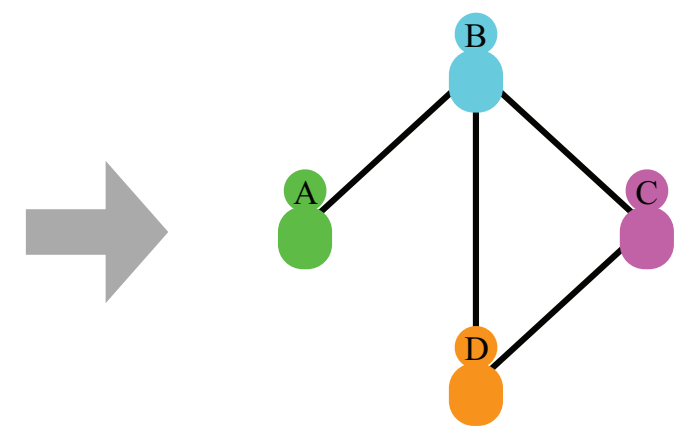

(A) Example temporal network with four individuals. (B) Aggregated static network corresponding to the temporal network shown in (A). The vertical lines show contacts between pairs of individuals.

long intervals between contacts). In many types of empirical data, the intervals between the contacts involving a certain pair of individuals follow a long-tailed distribution $[30,59,60]$, as shown in Fig. 3(A). Most such inter-contact times are relatively short, but a few are very long. In addition, successive inter-contact times are often positively correlated. In contrast, an implicit but crucial assumption underlying most theoretical and numerical analysis of epidemic dynamics in well-mixed populations and static networks is that inter-contact times obey an exponential distribution without correlation between different contacts. An example contact sequence generated under this assumption is shown in Fig. 3(B). The two sequences shown in Fig. 3 have the same number of contacts. The sequence shown in Fig. 3(B) lacks bursts, which contrasts with the sequence shown in Fig. 3(A), which shows the bursty nature of real temporal network data. The bursty nature of data may change the current understanding of epidemic dynamics on networks [61].

\section{Epidemiological models}

\section{The SIR model}

The mathematical epidemiological model that is probably the most widely used for theorizing about and emulating epidemics is the so-called Susceptible-Infected-Recovered (or, succinctly, the SIR) model [9,62-65]. In the individualbased version of the SIR model, with which we are concerned in the present report, each individual belongs to either a susceptible (S), infected (I), or recovered (R) state at any given time. The simplest version of the SIR model without demographic factors is defined as follows. When a susceptible individual and an infected individual interact, the former may be infected at an infection rate, denoted by $\beta$ (Fig. 4(A)). Precisely speaking, the susceptible individual contracts infection with probability $\beta \Delta t$ within a short time interval $\Delta t$. With probability $1-\beta \Delta t$, the susceptible individuals stays in the susceptible state throughout the interval $\Delta t$. $\Delta t$ can be identified with a discrete simulation time step. If a susceptible individual interacts with $k$ infected individuals at the same time, which is usually assumed in the case of epidemic simulations on static networks (see Fig. 4(B) for the case of $k=3$ ), the susceptible individual is infected with probability $1-(1-\beta \Delta t)^{k} \approx k$ $\beta \Delta t$ within small time $\Delta t$. The interval $\Delta t$ is chosen to be small enough to make this probability sufficiently small, to avoid the effect of time discretization. An infected individual is assumed to recover with rate $\mu$, irrespectively of the state (i.e., susceptible, infected, or recovered) of other individuals in the neighborhood. In other words, each infected individual transits to the recovered state with probability $\mu \Delta t$ within time $\Delta t$.

In the SIR model, " $\mathrm{R}$ " corresponds to the recovered state with immunity. Therefore, once an individual has entered the recovered state, the individual will never be infected again. When the initial population is a mixture of susceptible and 
Figure 3. Bursty nature of inter-contact times

\section{(A) Original}
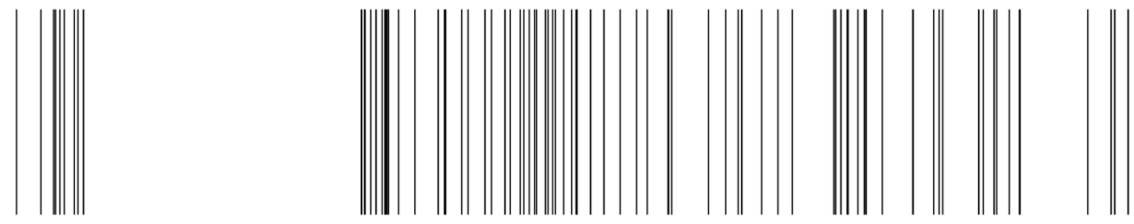

\section{(B) Exponential}

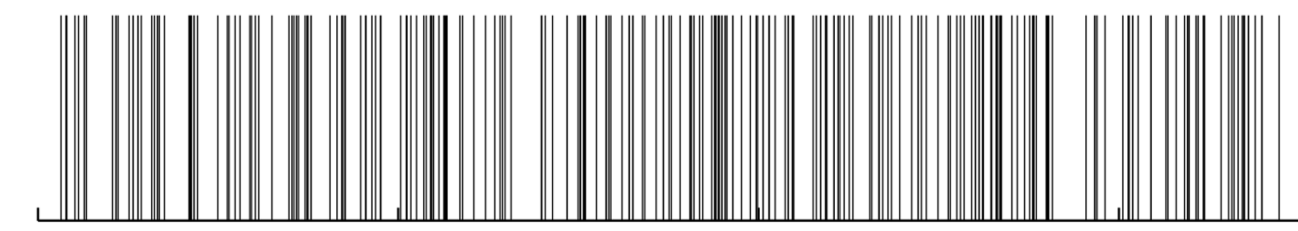

600

1000

1400

1800

\section{time (day)}

(A) Contact sequence that obeys a long-tail distribution. We generated it based on the behaviour of a sex buyer [25]. (B) Contact sequence whose inter-contact time distribution obeys an exponential distribution (i.e., Poisson process) with the same mean. The numbers of contacts in the two sequences are equal to each other.

Figure 4. SIR model

(A) infection recovery
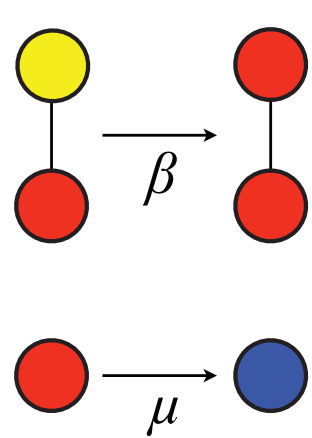

(B)

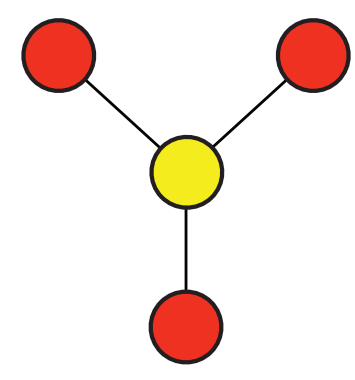

\section{susceptible (S) infected (I) recovered $(\mathrm{R})$}

(A) Schematic of infection and recovery in the SIR model. Infection occurs at rate $\beta$ per contact and recovery occurs at rate $\mu$. (B) The rate at which the susceptible individual is infected is proportional to the number of infected neighbors in the SIR model. 
infected individuals (usually the number of infected individuals is initially set to one for modelling purposes), the SIR dynamics stops with a mixture of susceptible and recovered individuals. Then, the final number of the recovered individuals is called the final (epidemic) size and represents the total damage to the population caused by an epidemic wave. Mathematically, the " $R$ " state can also signify death if the rate of encounters that an individual makes with other individuals is independent of the population size; the dead individual does not infect anybody and is not infected by anybody, which is functionally equivalent to a recovered and immune individual. For this reason, the SIR model is widely used to represent infectious diseases in which oneshot epidemic waves can occur and the mortality is high or the recovery implies immunity. Examples include influenza, SARS, measles, and mumps.

An important contribution of mathematical epidemiology to medicine is the concept of basic reproduction number and epidemic threshold $[5,9,64]$. The basic reproduction number, conventionally denoted by $R_{0}$, is the expected number of secondary infections of an infected individual in a population of all susceptible individuals. If $R_{0}$ exceeds unity, the epidemics can spread to a finite fraction of the population, meaning that epidemic spreading is a threshold phenomenon. The epidemic threshold is equivalent to $R_{0}=1$, where $R_{0}=\beta k / \mu$. Many studies have measured $R_{0}$ for different diseases and societies even though it is only directly related to an epidemic threshold in the context of the well-mixed SIR model. In temporal networks both the temporal structure and the network topology can move the threshold away from $R_{0}=1$.

\section{The SEIR model}

To explore more realistic models, one often uses extensions and variants of the SIR model, generally termed compartmental models $[5,9,64]$. One such example is the susceptible-exposed-infected-recovered (SEIR) model, whereby a new state " $\mathrm{E}$ " (for exposed) is added between susceptible and infected. The interaction between susceptible and infected individuals causes the former to transit to the latent exposed state. An exposed individual is infected but not infectious. After staying in the exposed state for some time, the exposed individual transits to the infected state to be able to infect others.

\section{The SI model}

For theoretical purposes, the susceptible-infected (SI) model is also used. The SI model is unrealistic in that an individual stays in the I state forever, but it captures the incipient stage of an outbreak. The SI model is devoid of the $\mathrm{R}$ state. Therefore, if there is at least one infected individual in the population, the SI dynamics necessarily ends up with the entire infected population. The SI model is used to investigate transient dynamics, in particular, the speed of infection in the initial and middle stages of epidemic spreading. For example, the analysis of the SI model on static networks revealed that the initial epidemic spreading is faster when the individuals have different numbers of contacts; (see Fig. 1 for an example) than when they all have the same number of contacts $[66,67]$. When infected individuals are rare in an early stage, the SI and SIR models behave almost the same because there are few recovered individuals.

\section{Epidemic spreading on temporal networks}

In this section, we describe the main findings regarding epidemic spreading on temporal networks. Explanation of this important topic has only recently started using a combination of real data, numerical simulations, and theory. A major research strategy for now is to compare the results (e.g. final size and spreading speed) obtained for temporal networks with those for randomized networks. Randomized networks are temporal or static networks generated by randomizing the original temporal networks, with some of the properties of the original networks conserved. A network obtained by randomizing the times of each contact is such an example. This randomization preserves the number of contacts on each link and the structure of the aggregated network, whereas it destroys the temporal structure of the contact sequence on each link. Randomized networks serve as null models. If epidemic spreading is different between the original and randomized networks, we can infer the properties of temporal networks that are responsible and those that are not responsible for the observed difference [2].

In the following, we will introduce the results obtained in the last few years. We note that the fact that temporal network effects influence epidemic spreading has been recognized longer than this, see e.g. [26,27,50,52,68-71]. However, systematically exploring different effects of temporal networks, or the corresponding aggregated networks, on epidemic spreading is a more recent theme. We survey some key studies that explored effects of temporal networks on the final size and time evolution of epidemic spreading. In order not to digress from the main points, we do not mention other aspects (e.g., temporal networks yield longer persistence of infection than the aggregated network in late stages of spreading [61,72-74]). We also briefly mention mathematical modelling (see [75] and references therein).

Karsai et al. numerically simulated the deterministic SI dynamics on the temporal network data of phone calls between people [76]. By the deterministic SI dynamics, we mean that in this model a contact between a pair of a susceptible individual and an infected individual always 
causes infection; this assumption reduces the number of parameters to be investigated. The mobile phone data contain about 4.5 million individuals, 9 million links, and 31 million events (i.e. phone calls). They compared transient spreading dynamics in temporal networks and that in randomized networks, whereby their different randomization methods destroy temporal structure of the data to different extents. The authors showed that epidemic spreading slows down on temporal networks compared with different randomized networks. They also corroborated their results with two other datasets, but without going into so much detail as the phone-call data. Similar conclusions are supported by their follow-up study [77], an approach based on the so-called average temporal path length [58], and different numerical simulations of the susceptible-infected-susceptible (SIS) model (whereby the recovered individual turns back to the susceptible state and is possibly reinfected, representing endemic diseases) on artificial temporal network data [78]. In contrast, Rocha et al. [79] observed that randomizing the time stamps sped up disease spreading in a network of sexual contacts between prostitutes and their sex buyers. The cause of this difference is still an open question. One hypothesis to be tested is that while the links of the dataset analysed in [76,77] are typically active throughout the sampling time, the links in Rocha et al.'s data last only for a short period (still, within this period, the contacts can be bursty as seen in Fig. 3(A)). An interesting general question is to identify temporal network structure that speeds up or slows down disease spreading. The results in [58], with a dataset different from the one analysed in $[76,77]$, also suggest that the temporal nature of networks enhances rather than suppresses epidemic spreading (figure $6 \mathrm{~b}$ in [58]). Also in a variant of the SI model, in which multiple infection attempts by infected individuals within a short time is necessary for a susceptible individual to be infected, epidemic spreading is more facilitated by empirical temporal structure compared to randomized reference data [80].

Miritello et al. numerically investigated stochastic SIR dynamics with a fixed recovery time on a mobile phone dataset containing 20 million individuals [37]. They observe that, when the per-contact infection probability (corresponding to infection rate $\beta$ shown in Fig. 4(A)) is large, the final size of the infected population for the temporal network data is smaller than that for the randomized temporal network, consistent with the results in [76]. Because of the bursty nature of the data (Fig. 3(A)), newly infected individuals have to wait longer for the next encounter with a susceptible individual, which opens a pathway for infection. Therefore, the authors conclude, a global outbreak is suppressed on this type of temporal network structure [37]. This mechanism should be
Figure 5. Correlated contact sequences on adjacent links

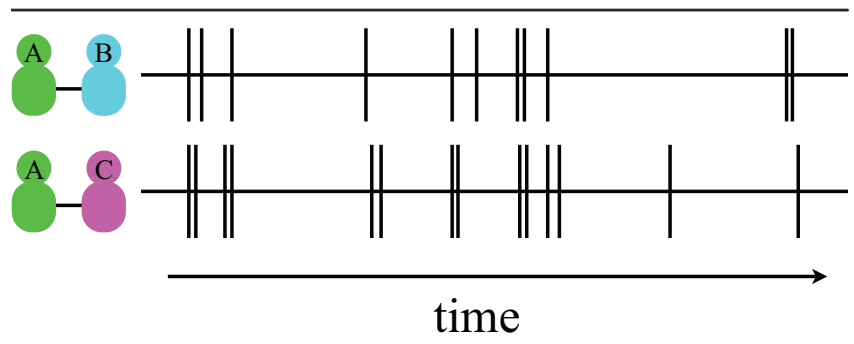

The contact times on two adjacent links (i.e., two links sharing an individual in this case "A") are often correlated with each other.

common to the system studied in [76]. In contrast, at small per-contact infection probabilities, where the final size of the infected population is presumably small, temporal networks yield a larger final size than the randomized networks do. Different numerical simulations with the SI model support similar conclusions [81]. Miritello et al. [37] conclude that group conversation contacts (i.e., correlations between the contact sequences of adjacent links-see Fig. 5-which are also treated in [77]) inherent in the data are responsible for the promotion of local contagion at a low per-contact infection probability. Because of the correlated occurrence of the contacts on adjacent links, a newly infected individual tends to meet a susceptible "prey" sooner in the original temporal network than in the case of the randomized networks.

Stehle et al. investigated stochastic SEIR epidemics on the temporal network recorded in a two-day medical conference [82]. They numerically showed that the final size of the infected population does not differ between the original temporal network and the aggregated network. The origin of the discrepancy in the results in different studies is unclear. Even mathematical analysis of epidemic spreading models with dynamic contacts (without using real data) yields opposite results. In a model based on the SIR dynamics, temporal dynamics of links speeds up epidemic spreading [83], whereas another SIS-based model with dynamic contact changes concludes the converse [84].

\section{Prevention of epidemic spreading using temporal networks}

One potentially important application of temporal network studies is to construct efficient immunization protocols. Faced with an evolving outbreak, society might only have resources to produce a limited amount of vaccine. This might not be such a big problem in practice because only a fraction of individuals in a population needs to be immunized for stopping epidemics (in other words, to achieve herd immunity). 
Mathematically we can phrase the problem as "how can we immunize a given fraction of the population to mitigate the disease as effectively as possible?" Two things are important to understand in coping with the immunization problem.

Firstly, in practice, we can only use local information. In other words, we can obtain information about specified individuals and their experience, but hardly map out all contacts of the entire population. In [85], Cohen et al. proposed an elegant method for static networks where one picks individuals at random, asks them to name an acquaintance that they meet regularly (such that the disease can be transmitted from one to the other), and immunize the acquaintance. With this procedure, so-called the neighbor immunization (sometimes, this procedure is known as ring vaccination, but this terminology is not always consistent), the probability of immunizing individuals with many network neighbors-obviously important for preventing disease spreading_increases.

Secondly, the immunization problem would benefit from accurately predicting future contacts and is therefore a temporal problem. Therefore, temporal information can be exploited in containing an outbreak. To this end, Lee et al. [86] extended the neighbor immunization protocol for static networks [85] to temporal networks. One of their protocols works by asking a randomly chosen individual to name the acquaintance that he or she met most recently. Lee et al. showed that this protocol outperforms the standard neighbor immunization on several empirical temporal networks [86].

In the context of mobile phone malware epidemics, Tang et al. examined a containment strategy based on temporal networks [87]. Their intervention method was to propagate a patch message, the reception of which immunized a mobile phone against the malware. In the context of infectious disease spreading of humans, this protocol would be analogous to word-of-mouth type information spreading combined with voluntary means of lowering the probability of acquiring the infection, such as vaccination, pre-exposure prophylaxis [88], use of condoms, and hand washing. They showed that the so-called temporal closeness centrality, which is a score calculated for each node and intuitively quantifies the abundance of epidemic pathways from/to the node, is an efficient measure for prioritizing the mobile phones into which the patch message is injected.

We are not aware of other immunization methods that exploit both the network topology and the temporal structure of contacts. As the studies mentioned above suggest, exploiting temporal information likely leads to improvement of network-based immunization protocols. This idea is currently an understudied question. Various link-prediction techniques, in which occurrence of contacts in the future is predicted based on the temporal network data at hand [89-91], may offer a promising approach to this problem.

\section{What does the future hold?}

The results of studying disease spreading and its prevention on temporal networks seem to be model dependent. In addition, they are likely to also depend on datasets. So far, most studies have employed different models and datasets and this complicates the comparison between different studies and any comprehensive understanding of them. To better understand and intervene in epidemic spreading using temporal networks, we need a more systematic comparison across different epidemic models and datasets. To this end, platforms that make network data available for academic researchers are very valuable. Some projects generously make their data, including temporal network data, open for academic use (e.g. Socio Patterns project http://www.sociopatterns.org/datasets/ and Stanford Large Network Data Collection http://snap. stanford.edu/data/).

Other future challenges include exploring the intersection between temporal networks and metapopulation networks. The metapopulation network is a modeling framework in which a node (called the metapopulation) in a network is a place (like home, school, or workplace) rather than an individual. Individuals move from one metapopulation to another, and at the same time, disease transmission can occur between individuals in the same metapopulation. Such a framework has been investigated in mathematical epidemiology for a couple of decades $[64,92,93]$, and more recent network epidemiology has deepened the results and also established strong linkage between data and models [15]. This framework was also used by the GLEAMviz project to predict the H1N1 pandemic [16]. Human travel certainly has temporal components [33, 94], and links of metapopulation networks may also be dynamic, but on a slower timescale [49]. A recent study reports that introduction of dynamics to links changes the final size of the SIR model on metapopulation networks [95]. Applying temporal network analysis of epidemic spreading to metapopulation networks may thus be a fruitful direction to take.

Related to the intersection between the metapopulation framework and temporal networks is the analysis of the effects of travel restriction. A majority of such studies, many of which are based on metapopulation networks, suggest that travel restriction is a poor strategy to contain epidemic outbreaks [96-103]. There are, however, 
dissonant results that find travel restriction effective $[103,104]$. Traveling is a highly temporal phenomenon, such that travel restriction implemented at right moments of time may be effective in containing epidemic spreading.

It may also be an important question to determine the equivalent of the basic reproduction number $R_{0}$ and epidemic threshold for temporal networks. It is recently debated that $R_{0}$ is misused in situations to which its original definition does not apply or when its reliable estimation is difficult in practice [65,105-107]. Another interesting question may be what the traditional basic reproduction number tells us about outbreaks in temporal networks. Even more fundamental perhaps, it would be interesting to examine how much improvement temporal-network methods can make on staticnetwork models in fitting to empirical outbreak data.

Another challenge for applications is to identify specific pathogens and situations for which temporal network approaches are useful. Although social contacts are dynamic in general, analytical and computational methods for static network epidemiology are more powerful than those for temporal networks. Therefore, we can be content with results derived from static network epidemiology in the limit of slow network dynamics relative to the epidemic dynamics. In contrast, typical childhood diseases such as mumps, measles, and pertussis can transmit infection upon short and nonintense contacts between individuals, such that descriptions under the temporal network framework may be useful. Another possible example is foot-and-mouth disease, which is highly contagious among livestocks [108]. Contact patterns of livestocks such as cattle and sheep form temporal networks because they move among premises because of trading [50-53]. It could furthermore be interesting to study improvements of medical practice that today operate on the contact network, such as contact tracing (where individuals testing positive for a pathogen have to name their recent contacts so that they can be called for testing). Perhaps temporal-network methods can give improved estimates of how far back in time it is necessary to pursue the tracing.

\section{Abbreviations \\ SEIR, susceptible-exposed-infected-recovered; SI, susceptible-infected; SIR, susceptible-infected-recovered; SIS, susceptible-infected-susceptible; STI, sexually trans- mitted infection.}

\section{Disclosure}

The authors declare that they have no disclosures.

\section{Acknowledgements}

We acknowledge Taro Takaguchi for critical reading of the manuscript. We acknowledge financial supports provided through Grants-in-Aid for Scientific Research (No. 23681033) from MEXT, Japan (NM) the Swedish Research Council and the WCU program through NRF Korea funded by MEST R31-2008-10029-0 (PH).

\section{References}

I. Kempe D, Kleinberg J, Kumar A: Connectivity and inference problems for temporal networks. Proc STOC 2000:504-I3.

2. Holme P, Saramäki J: Temporal networks. Phys Rep 2012, 519:97-I25.

3. Albert R, Barabási A-L: Statistical mechanics of complex networks. Rev Mod Phys 2002, 74:47-97.

4. Barabási A-L: Linked - The New Science of Networks. Cambridge: Perseus Publishing; 2002.

5. Giesecke J: Modern Infectious Disease Epidemiology. 2nd edition. London: Arnold; 2002.

6. Newman MEJ: The structure and function of complex networks. Siam Rev 2003, 45: 167-256.

7. Keeling MJ, Eames KTD: Networks and epidemic models. J R Soc Interface 2005, 2:295-307.

FlOOOPrime

RECOMMENDED

8. May RM: Network structure and the biology of populations. Trends Ecol Evol 2006, 21 :394-9.

\section{FlOOOPrime}

\section{RECOMMENDED}

9. Keeling MJ, Rohani P: Modeling Infectious Diseases in Humans and Animals. Princeton: Princeton University Press; 2007.

10. Riley S: Large-scale spatial-transmission models of infectious disease. Science 2007, 3 |6:|298-|30|.

\section{FlOOOPrime}

RECOMMENDED

II. Barrat A, Barthélemy M, Vespignani A: Dynamical Processes on Complex Networks. Cambridge: Cambridge University Press; 2008.

12. Cohen R, Havlin S: Complex Networks - Structure, Robustness and Function. Cambridge: Cambridge University Press; 2010.

13. Easley D, Kleinberg J: Networks, Crowds, and Markets - Reasoning about a Highly Connected World. Cambridge: Cambridge University Press; 2010.

14. Newman MEJ: Networks - An Introduction. Oxford: Oxford University Press; 2010.

15. Vespignani A: Modelling dynamical processes in complex sociotechnical systems. Nat Phys 2012, 8:32-9.

16. Balcan D, Hu H, Goncalves B, Bajardi P, Poletto C, Ramasco JJ, Paolotti D, Perra N, Tizzoni M, Van den Broeck W, Colizza V, Vespignani A: Seasonal transmission potential and activity peaks of the new influenza $A(H I N I)$ : a Monte Carlo likelihood analysis based on human mobility. BMC Medicine 2009, 7:45.

FlOOOPrime RECOMMENDED

17. Van den Broeck W, Gioannini C, Gonçalves B, Quaggiotto M, Colizza V, Vespignani A: The GLEaMviz computational tool, a publicly available software to explore realistic epidemic spreading scenarios at the global scale. BMC Infect Dis 201 I, I I:37.

\section{FlOOOPrime} RECOMMENDED

18. Bajardi P, Poletto C, Balcan D, Hu H, Goncalves B, Ramasco J], Paolotti D, Perra N, Tizzoni $M$, Van den Broeck W, Colizza V, Vespignani A: Modeling vaccination campaigns and the Fall/Winter 2009 
activity of the new $A(H I N I)$ influenza in the Northern Hemisphere. Emerg Health Threats J 2009, 2:el I.

19. Haraldsdottir S, Gupta S, Anderson RM: Preliminary studies of sexual networks in a male homosexual community in Iceland. J Acquir Immune Defic Syndr 1992, 5:374-8I.

20. Liljeros F, Edling CR, Amaral LAN, Stanley HE, Åberg Y: The web of human sexual contacts. Nature 200 I, 4 I I:907-8.

\section{FlOOOPRime
RECOMMENDED}

21. Liljeros F, Edling CR, Amaral LAN: Sexual networks: implications for the transmission of sexually transmitted infections. Microbes Infect 2003, 5:189-96.

22. Bearman PS, Moody J, Stovel K: Chains of affection: the structure of adolescent romantic and sexual networks. Am J Sociol 2004, | | 0:44-9|.

\section{FlOOOPrime}

RECOMMENDED

23. Dietz K, Hadeler KP: Epidemiological models for sexually transmitted diseases. J Math Biol 1988, 26: I-25.

24. Watts $\mathrm{CH}$, May RM: The influence of concurrent partnerships on the dynamics of HIVIAIDS. Math Biosci 1992, 108:89-104.

25. Rocha LEC, Liljeros F, Holme P: Information dynamics shape the sexual networks of Internet-mediated prostitution. $P$ Natl Acad Sci USA 2010, 107:5706-II.

26. Morris M, Kretzschmar M: Concurrent partnerships and transmission dynamics in networks. Soc Netw 1995, 17:299-318.

\section{FIOOOPrime \\ RECOMMENDED}

27. Kretzschmar M, Morris M: Measures of concurrency in networks and the spread of infectious disease. Math Biosci 1996, I33:165-95.

\section{FlOOOPrime \\ RECOMMENDED}

28. Danowski JA, Edison-Swift P: Crisis effects on intraorganizational computer-based communication. Comm Res 1985, I 2:25I-70.

29. Balthrop J, Forrest S, Newman MEJ, Williamson MM: Technological networks and the spread of computer viruses. Science 2004, 304:527-9.

30. Eckmann J-P, Moses E, Sergi D: Entropy of dialogues creates coherent structures in e-mail traffic. P Natl Acad Sci USA 2004, I0I:14333-7.

31. Villani A, Frigessi A, Liljeros F, Nordvik MK, de Blasio BF: A characterization of Internet dating network structures among Nordic men who have sex with men. PloS one 2012, 7:e39717.

32. Onnela J-P, Saramäki J, Hyvönen J, Szabó G, Lazer D, Kaski K, Kertész J, Barabási A-L: Structure and tie strengths in mobile communication networks. P Natl Acad Sci USA 2007, 104:7332-6.

33. González MC, Hidalgo CA, Barabási A-L: Understanding individual human mobility patterns. Nature 2008, 453:779-82.

34. Wang P, González MC, Hidalgo CA, Barabási A-L: Understanding the spreading patterns of mobile phone viruses. Science 2009 , 324: $107 \mid-6$

35. Song C, Qu Z, Blumm N, Barabási A-L: Limits of predictability in human mobility. Science 2010, 327:1018-2I.

36. Jo H-H, Pan RK, Kaski K: Emergence of bursts and communities in evolving weighted networks. PloS one 20II, 6:e22687.

37. Miritello G, Moro E, Lara R: Dynamical strength of social ties in information spreading. Phys Rev E 20II, 83:045 I02(R).

\section{FlOOOPrime}

38. Eagle N, Pentland AS: Reality mining: sensing complex social systems. Pers Ubiquit Comput 2006, 10:255-68.

39. Kim T, ChangA, Holland L, Pentland AS: Meeting mediator: enhancing group collaboration using sociometric feedback. Proc 2008 ACM Conference on Computer Supported Cooperative Work 2008, 457-66.
40. Wu L, Waber B, Aral S, Brynjolfsson E, Pentland AS: Mining face-toface interaction networks using sociometric badges: predicting productivity in an it configuration task. Proc International Conference on Information Systems 2008, 14-7.

4I. Cattuto C, Van den Broeck W, Barrat A, Colizza V, Pinton J-F, Vespignani $A$ : Dynamics of person-to-person interactions from distributed RFID sensor networks. PloS one 2010, 5:el I596.

\section{FlOOOPrime \\ RECOMMENDED}

42. Isella L, Stehlé J, Barrat A, Cattuto C, Pinton J-F, Van den Broeck W: What's in a crowd? Analysis of face-to-face behavioral networks. J Theor Biol 201 I, 271:166-80.

\section{FIOOOPrime}

43. Yano K, Ara K, Moriwaki N, Kuriyama H: Measurement of human behavior: creating a society for discovering opportunities. Hitachi Review 2009, 58:139-44.

44. Wakisaka Y, Ara K, Hayakawa M, Horry Y, Moriwaki N, Ohkubo N, Sato N, Tsuji S, Yano K: Beam-scan sensor node: reliable sensing of human interactions in organization. Proc $6^{\text {th }}$ International Conference on Networked Sensing Systems 2009:58-6I.

45. Takaguchi T, Nakamura M, Sato N, Yano K, Masuda N: Predictability of conversation partners. Phys $\operatorname{Rev} X 201 \mathrm{I}$, I:01 1008.

46. Takaguchi T, Sato N, Yano K, Masuda N: Importance of individual events in temporal networks. New J Phys 2012, 14:093003.

47. Isella L, Romano M, Barrat A, Cattuto C, Colizza V, Van den Broeck W, Gesualdo F, Pandolfi E, Ravà L, Rizzo C, Tozzi AE: Close encounters in a pediatric ward: measuring face-to-face proximity and mixing patterns with wearable sensors. PloS one 201 I, 6: article No. el7|44.

48. Stehlé J, Voirin N, Barrat A, Cattuto C, Isella L, Pinton J-F, Quaggiotto M, Van den Broeck W, Régis C, Lina B, Vanhems P: High-resolution measurements of face-to-face contact patterns in a primary school. PloS one 2011, 6:e23176.

49. Gautreau A, Barrat A, Barthélemy M: Microdynamics in stationary complex networks. P Natl Acad Sci USA 2009, 106:8847-52.

\section{FlOOOPrime} RECOMMENDED

50. Kao RR, Green DM, Johnson J, Kiss IZ: Disease dynamics over very different time-scales: foot-and-mouth disease and scrapie on the network of livestock movements in the UK. J R Soc Interface 2007, 4:907-16.

51. Heath MF, Vernon MC, Webb CR: Construction of networks with intrinsic temporal structure from UK cattle movement data. BMC Vet Res 2008, 4: article No. II.

52. Vernon MC, Keeling M]: Representing the UK's cattle herd as static and dynamic networks. P R Soc B 2009, 276:469-76.

53. Bajardi P, Barrat A, Natale F, Savini L, Colizza V: Dynamical patterns of cattle trade movements. PloS one 20II, 6: article No. el 9869.

54. Blonder B, Dornhaus A: Time-ordered networks reveal limitations to information flow in ant colonies. PloS one 2011, 6: article No. e20298.

55. Tantipathananandh C, Berger-Wolf T, Kempe D: A framework for community identification in dynamic social networks. Proc. KDD’07 2007:7।7-26.

56. Holme P: Network reachability of real-world contact sequences. Phys Rev E 2005, 71: article No. 046I I 9.

57. Tang J, Musolesi M, Mascolo C, Latora V: Temporal distance metrics for social network analysis. Proc. WOSN'09 2009:3 I-6.

58. Pan RK, Saramäki J: Path lengths, correlations, and centrality in temporal networks. Phys Rev E 20II, 84:016I05.

59. Barabási A-L: The origin of bursts and heavy tails in human dynamics. Nature 2005, 435:207-II. 
60. Vázquez A, Oliveira JG, Dezsö Z, Goh K-I, Kondor I, Barabási A-L: Modeling bursts and heavy tails in human dynamics. Phys Rev $E$ 2006, 73: article No. 036I27.

6I. Vazquez A, Rácz B, Lukács A, Barabási A-L: Impact of nonPoissonian activity patterns on spreading processes. Phys Rev Lett 2007, 98:158702

62. Kermack WO, McKendrick AG: A contribution to the mathematical theory of epidemics. P R Soc Lond A I927, I I 5:700-2 I.

63. Bailey NTJ: The simulation of stochastic epidemics in two dimensions. Proc 5th Berkeley Symposium on Mathematical Statistics and Probability 1967, 4:237-7.

64. Anderson RM, May RM: Infectious Diseases of Humans - Dynamics and Control. Oxford: Oxford University Press; 199I.

65. Roberts MG: The pluses and minuses of $\boldsymbol{R}_{\mathbf{0}}$. J R Soc Interface 2007, 4:949-6I.

66. Barthélemy M, Barrat A, Pastor-Satorras R, Vespignani A: Velocity and hierarchical spread of epidemic outbreaks in scale-free networks. Phys Rev Lett 2004, 92: I78701.

\section{FlOOOPrime} RECOMMENDED

67. Barthélemy M, Barrat A, Pastor-Satorras R, Vespignani A: Dynamical patterns of epidemic outbreaks in complex heterogeneous networks. J Theor Biol 2005, 235:275-88.

68. Eames KTD, Keeling MJ: Monogamous networks and the spread of sexually transmitted diseases. Math Biosci 2004, I 89: I I5-30.

69. Fefferman $\mathrm{NH}, \mathrm{Ng} \mathrm{KL}$ : How disease models in static networks can fail to approximate disease in dynamic networks. Phys Rev E 2007, 76: article No. 031919.

70. Volz E, Meyers LA: Susceptible-infected-recovered epidemics in dynamic contact networks. P R Soc B 2007, 274:2925-33.

\section{FlOOOPrime} RECOMMENDED

7I. Smieszek T, Fiebig L, Scholz RW: Models of epidemics: when contact repetition and clustering should be included. Theor Biol Med Model 2009, 6: article No. II.

72. Iribarren JL, Moro E: Impact of human activity patterns on the dynamics of information diffusion. Phys Rev Lett 2009, I03: article No. 038702.

73. Karrer B, Newman MEJ: Message passing approach for general epidemic models. Phys Rev E 2010, 82:016101.

74. Min B, Goh K-I, Vazquez A: Spreading dynamics following bursty human activity patterns. Phys Rev E 201 I, 83:036I02.

75. Bansal S, Read J, Pourbohloul B, Meyers LA: The dynamic nature of contact networks in infectious disease epidemiology. J Biol Dyn 2010, 4:478-89.

\section{FlOOOPrime}

\section{RECOMMENDED}

76. Karsai M, Kivelä M, Pan RK, Kaski K, Kertész J, Barabási A-L, Saramäki J: Small but slow world: how network topology and burstiness slow down spreading. Phys Rev E 2011, 83: article No. 025I02(R).

\section{FlOOOPrime}

\section{RECOMMENDED}

77. Kivelä M, Pan RK, Kaski K, Kertész J, Saramäki J, Karsai M: Multiscale analysis of spreading in a large communication network. J Stat Mech 2012, 3:P03005.

78. Perra N, Gonçalves B, Pastor-Satorras R, Vespignani A: Activity driven modeling of time varying networks. Sci Rep 2012, 2: article No. 469.

79. Rocha LEC, Liljeros F, Holme P: Simulated epidemics in an empirical spatiotemporal network of 50,185 sexual contacts. Plos Comput Biol 201 I, 7: article No. el00I 109.

80. Takaguchi T, Masuda N, Holme P: Bursty communication patterns facilitate spreading in a threshold-based epidemic dynamics. Preprint 2012 Jun: arXiv: | 206.2097.v I [http://arxiv.org/abs/ I206.2097]

8I. Rocha LEC, Decuyper A, Blondel VD: Epidemics on a stochastic model of temporal network. Preprint 20 I 2 Mar: arXiv: | 204.542 I.v | [http://arxiv.org/abs/I204.542I]

82. Stehlé J, Voirin N, Barrat A, Cattuto C, Colizza V, Isella L, Régis C, Pinton J-F, Khanafer N, Van den Broeck W, Vanhems P: Simulation of an SEIR infectious disease model on the dynamic contact network of conference attendees. BMC Medicine 20I I, 9: article No. 87.

83. Volz E, Meyers LA: Epidemic thresholds in dynamic contact networks. J R Soc Interface 2009, 6:233-4I

84. Risau-Gusman S: Influence of network dynamics on the spread of sexually transmitted diseases. J R Soc Interface 20 I2, 9: I 363-72.

85. Cohen R, Havlin S, ben-Avraham D: Efficient immunization strategies for computer networks and populations. Phys Rev Lett 2003, 91: article No. 24790I.

\section{FlOOOPrime
RECOMMENDED}

86. Lee S, Rocha LEC, Liljeros F, Holme P: Exploiting temporal network structures of human interaction to effectively immunize populations. PloS one 2012, 7: article No. e36439.

87. Tang J, Mascolo C, Musolesi M, Latora V: Exploiting temporal complex network metrics in mobile malware containment. Presented at Proc IEEE International Symposium on a World of Wireless, Mobile and Multimedia Networks (WoWMoM) 201 I:I-9 June 20II; Lucca, Italy.

88. Grant RM, Lama JR, Anderson PL, McMahan V, Liu AY, Vargas L, Goicochea P, Casapía M, Guanira-Carranza JV, Ramirez-Cardich ME, Montoya-Herrera O, Fernández T, Veloso VG, Buchbinder SP, Chariyalertsak S, Schechter M, Bekker L-G, Mayer KH, Kallás EG, Amico KR, Mulligan K, Bushman LR, Hance RJ, Ganoza C, Defechereux P, Postle B, Wang F, McConnell JJ, Zheng J-H, Lee J, et al.: Preexposure chemoprophylaxis for HIV prevention in men who have sex with men. $N$ Engl J Med 2010, 363:2587-99.

\section{FlOOOPrime
RECOMMENDED}

89. Liben-Nowell $D$, Kleinberg J: The link-prediction problem for social networks. J Am Soc Inf Sci Tec 2007, 58: I019-31.

90. Hasan MA, Zaki MJ: A survey of link prediction in social networks. Soc Netw Data Anal 20 I I:243-75.

91. Lü L, Zhou T: Link prediction in complex networks: a survey. Physica A 20II, 390: II50-70.

92. Rvachev LA, Longini IM Jr: A mathematical model for the global spread of influenza. Math Biosci 1985, 75:3-22.

93. Diekmann O, Heesterbeek JAP, Metz JAJ: On the definition and the computation of the basic reproduction ratio $R_{0}$ in models for infectious diseases in heterogeneous populations. J Math Biol 1990, 28:365-82.

94. Brockmann D, Hufnagel L, Geisel T: The scaling laws of human travel. Nature 2006, 439:462-5.

\section{FlOOOPrime}

\section{RECOMMENDED}

95. Liu S, Baronchelli A, Perra N: Contagion dynamics in timevarying metapopulation networks. Preprint 2012 Oct: arXiv: I 2 |0.2776.vI [http://arxiv.org/abs/I2 I0.2776]

96. Cooper BS, Pitman RJ, Edmunds WJ, Gay NJ: Delaying the international spread of pandemic influenza. PLoS Med 2006, 3:e2 12.

97. Ferguson NM, Cummings DAT, Fraser C, Cajka JC, Cooley PC, Burke DS: Strategies for mitigating an influenza pandemic. Nature 2006, 442:448-52.

98. Hollingsworth TD, Ferguson NM, Anderson RM: Will travel restrictions control the international spread of pandemic influenza? Nat Med 2006, I 2:497-9. 
99. Colizza V, Barrat A, Barthelemy M, Valleron A-J, Vespignani A: Modeling the worldwide spread of pandemic influenza: Baseline case and containment interventions. PLoS Med 2007, 4:e I3.

100. Epstein JM, Goedecke DM, Yu F, Morris RJ, Wagener DK, Bobashev GV: Controlling pandemic flu: the value of international air travel restrictions. PloS one 2007, 2:e40I.

I0I. Colizza V, Vespignani A: Epidemic modeling in metapopulation systems with heterogeneous coupling pattern: theory and simulations. J Theor Biol 2008, 25 I:450-67.

102. Bajardi P, Poletto C, Ramasco JJ, Tizzoni M, Colizza V, Vespignani A: Human mobility networks, travel restrictions, and the global spread of 2009 HINI pandemic. PloS one 20II, 6:el659I.

103. Wang B, Cao L, Suzuki H, Aihara K: Safety-information-driven human mobility patterns with metapopulation epidemic dynamics. Sci Rep 2012, 2:887.
104. Brownstein JS, Wolfe CJ, MandI KD: Empirical evidence for the effect of airline travel on inter-regional influenza spread in the United States. PLoS Med 2006, 3:e40I

\section{FlOOOPrime}

RECOMMENDED

105. Heffernan JM, Smith RJ, Wahl LM: Perspectives on the basic reproductive ratio. $J R$ Soc Interface 2005, 2:28I-93.

106. Wallinga J, Lipsitch M: How generation intervals shape the relationship between growth rates and reproductive numbers. $P R$ Soc B 2007, 274:599-604.

107. Li J, Blakeley D, Smith RJ: The failure of $\boldsymbol{R}_{\mathbf{0}}$. Comput Math Methods Med 2011, 201 I:527610.

108. Haydon DT, Kao RR, Kitching RP: The UK foot-and-mouth disease outbreak - the aftermath. Nat Rev Micro 2004, 2:675-8I. 